\title{
Modification of Procedure to Estimate Viscosity of Bimodal Alumina/Epoxy Mixtures during a Centrifugal Process
}

\author{
Noriyuki Hayashi* ${ }^{*}$ Member \\ Wibowo Sulistio ${ }^{*} \quad$ Member \\ Shin-ichiro Tsuru ${ }^{* *}$ Member \\ Masanori Hara $^{* * *}$ Member
}

\begin{abstract}
The coefficient of viscosity $\eta$ is one of the major parameters to govern the movement of particles in alumina/epoxy mixtures during the centrifugal process. During the centrifugal process, the particle fraction and corresponding values of $\eta$ at a certain point varies spatiotemporally. In the present contribution, theoretical consideration to explain dependency of $\eta_{b}$ of bimodal alumina/epoxy mixtures on particle fractions is conducted. It is found that $\eta_{b}$ can be estimated as a function of two $\eta$ 's obtained for the unimodal mixtures that contain the fine and coarse particles, respectively. A modification about the definition of the coarse particle fraction from the conventional method was successfully introduced, resulting in better approximation of $\eta_{b}$ under the conditions of high particle fractions.
\end{abstract}

Keywords: functionally graded material, alumina/epoxy mixture, viscosity, bimodal system, centrifugal method

\section{Introduction}

Functionally graded materials (FGMs) are a new class of materials in which the composition and/or the microstructure spatially varies. They have promising potentials in the development of new solid insulation materials for future power apparatus ${ }^{(1)(2)}$. The authors are using a centrifugal method to fabricate FGM's with large size from alumina-particles/epoxy-resin mixtures (3). During the centrifugal process, the particle fraction at a certain location in the mixture spatiotemporally varies from almost zero to several tens percent. Consequently, $\eta$ in the mixture, which is a function of the particle fraction, varies spatiotemporally.

In this contribution, we proposed a modification of the conventional method (i.e., Farris equation) to obtain better estimation of $\eta$ for bimodal mixtures with high particle fraction.

\section{Materials and Experimental Method}

Spherical alumina particles with nominal diameters of $35 \mu \mathrm{m}$ and $80 \mu \mathrm{m}$ were employed as fine and coarse particles, respectively, and they were mixed with an epoxy resin. Description of both particle property and method of measurement can be seen in our previous papers ${ }^{(4)(5)}$.

\section{Results and Discussions}

When the fine and coarse particles by the volume of $V_{f}$ and $V_{c}$, respectively, were mixed with an epoxy resin by the volume of $V_{e}$, two kinds of the volume fraction of particles can be defined: one is ratio of the volume of all particles to the volume of the mixture

* ASEM, Faculty of Engineering Sciences, Kyushu University 6-1, Kasuga Kohen, Kasuga 816-8580

** Research Lab., Kyushu Electric Power Co., Inc. 2-1-47, Shiobaru, Minami-ku, Fukuoka 815-8520

*** EES, Faculty of Information Sciences and Electrical Engineering, Kyushu University

6-10-1, Hakozaki, Higashi-ku, Fukuoka 816-8580
$\nu_{p}=\left(V_{f}+V_{c}\right) /\left(V_{f}+V_{c}+V_{e}\right)$, and the other is ratio of the volume of the coarse particles to the volume of all particles $\nu_{\text {coarse }}=V_{c} /\left(V_{f}+V_{c}\right)$.

3.1 Experimental results Symbols in Fig. 1 show measured values of $\eta_{b}$ as a function of $\nu_{c}$ obtained for various $\nu_{p}{ }^{(5)} . \nu_{p}$ varied between 0 to $45 \%$, and $\nu_{\text {coarse }}$ varied between 0 to $100 \%$ for each $\nu_{p}$. It is noted that the bimodal mixtures with $\nu_{\text {coarse }}=0 \%$ and $100 \%$ correspond to the unimodal mixtures with the fine and coarse particles only, respectively.

3.2 Discussions A procedure proposed by Farris ${ }^{(6)}$ can be applied to approximate $\eta_{b}$ of the bimodal mixture from two $\eta_{u}$ 's, each of them is corresponding to the unimodal mixture each component contained in the bimodal mixture, and is given by the following expression.

$$
\eta_{b}\left(\nu_{p}, \nu_{\text {coarse }}\right)=\eta_{0} \cdot \frac{\eta_{f}\left(\nu_{f}\right)}{\eta_{0}} \cdot \frac{\eta_{c}\left(\nu_{c}\right)}{\eta_{0}} \cdots \ldots \ldots
$$

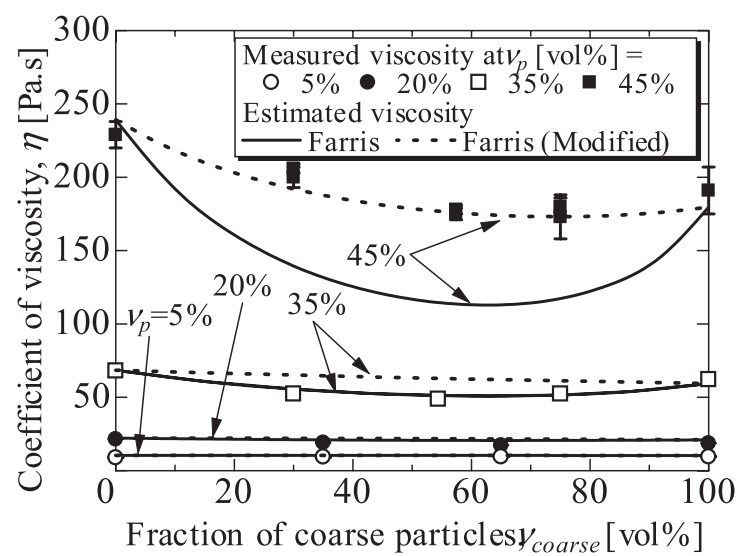

Fig. 1. $\eta_{b}$ of bimodal mixtures as a function of $\nu_{\text {coarse }}$ for various $\nu_{p}$; Symbols: Measured, Solid lines: Estimated 
Here, $\eta_{f}\left(\nu_{f}\right)$ and $\eta_{c}\left(\nu_{c}\right)$ are $\eta$ of the unimodal mixtures involving the fine and coarse particles, respectively, and are estimated by using the following Brinkman equa$\operatorname{tion}^{(7)}$ :

$$
\eta_{i}\left(\nu_{i}\right)=\eta_{0} /\left(1-\frac{\nu_{i}}{\nu_{i, \max }}\right)^{n} \ldots \ldots \ldots \ldots \ldots \ldots
$$

where, suffix $i=f$ and $c$ for the fine and coarse particles, respectively, $\eta_{0}$ is the coefficient of viscosity of the epoxy resin, $\nu_{i, \max }$ is the maximum packing fraction obtainable for the mixtures under consideration, and $n$ is a constant. In general cases, $n=2.5$ can be used with reasonable accuracy.

For the bimodal mixture under consideration, dependency of $\eta_{b}$ on $\nu_{\text {coarse }}$ were calculated using Eq. 1 for $\nu_{p}=5,20,35,45 \%$, and the results are plotted by solid lines in Fig. 1. In this calculation, $\nu_{f}$ and $\nu_{c}$ are calculated by the following equations, respectively:

$$
\begin{aligned}
& \nu_{f}=\frac{V_{f}}{V_{e}+V_{f}} \cdots \\
& \nu_{c}=\frac{V_{c}}{V_{e}+V_{f}+V_{c}}
\end{aligned}
$$

It is obvious from comparison between the measured and estimated values shown in Fig. 1 that the approximation procedure yields reasonable estimation of $\eta_{b}$ for $\nu_{p}$ smaller than $35 \%$, while it underestimates $\eta_{b}$ for $\nu_{p}$ larger than $45 \%$.

To improve the accuracy of estimation of $\eta_{b}$ for large $\nu_{p}$, a modification to the definition of the coarse particle fraction given by Eq. 4 was carried out. Dotted lines in Fig. 1 are obtained by applying the same Eq. 1 as the solid lines, except that a new definition of the packing fraction $\nu_{c}^{\prime}$ of coarse particles given by Eq. 5 is introduced instead.

$$
\nu_{c}^{\prime}=\frac{V_{c}}{V_{e}+V_{c}}
$$

Comparison between the measured and estimated values shown in Fig. 1 indicates that the new method based on Eq. 1 using the new definition of $\nu_{c}^{\prime}$ given by Eq. 5 gives reasonable estimation of $\eta_{b}$ for $\nu_{p}$ larger than $45 \%$.

\section{Conclusions}

The modification of Farris method proposed in this contribution enables better approximation of $\eta_{b}$ of the bimodal mixture for higher packing fraction larger than $45 \%$. The combination of this modification and the original Farris method enables the approximation of the spatiotemporally varying $\eta_{b}$ during the centrifugal process.

\section{Acknowledgment}

This work is partly supported by Grant-in-aids for Scientific Research (C)(2) \#16560244, and Joint research project on FGM between Kyushu University, Kyushu Electric Power Co., Inc., and Nishi Nippon Wire \& Cable Co., Inc.

(Manuscript received April 25, 2005)

\section{References}

(1) N. Hayashi, M. Sumikura, and M. Hara: "Numerical Analysis of Surface Electric Field Profiles for Spacers with Continuous Grading of Permittivity", Engineering Sciences Report, Kyushu University, Vol.24, No.1, pp.1-8 (2002-6)

( 2 ) K. Kato, K. Kimura, S. Sakuma, and H. Okubo: "Functionally Gradient Materials (FGM) for GIS Spacer Insulation", Proc. 12th ISH, Vol.2, pp.401-404 (2001)

( 3 ) N. Hayashi, S.I. Tsuru, T. Onoda, Y. Sakamoto, and M. Hara: "Fabrication of Functionally Graded Epoxy Resin with Alumina Fillers and Its Simulation by Using a Centrifugal Method", IEEJ Trans. FM, Vol.124, No.7, pp.598-606 (20047)

(4) N. Hayashi, T. Onoda, Y. Sakamoto, S.I. Tsuru, T. Kawabe, and M. Hara: "Fabrication of Permittivity Graded Epoxy Resin with Non-Uniform Dispersion of Alumina Fillers by Centrifugal Procedure", Materials Science Forum, Vol.492493, pp.501-506 (2005)

(5) N. Hayashi, W. Sulistio, S.I. Tsuru, T. Onoda, Y. Sakamoto, and M. Hara: "Viscosity of Unimodal and Bimodal Alumina/Epoxy Mixtures", IEEJ Trans. FM, Vol.125, No.3, pp.272-273 (2005-3)

(6) R.J. Farris: "Prediction of the viscosity of Multimodal Suspensions from Unimodal Viscosity Data", Trans. Soc. Rheology, 12:2, pp. 281-301 (1968)

( 7 ) H.C. Brinkman: "The Viscosity of Concentrated Suspensions and Solutions", J. Chem. Phys., Vol.20, No.4, p.571 (1952)

Noriyuki Hayashi (Member) is an Associate Professor of

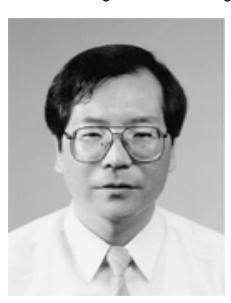
Faculty of Engineering Sciences, Kyushu University. He is engaged in the study of application of FGM to power engineering and the electromagnetic environmental problems associated with power systems. He is a member of the Institute of Electrical Engineers of Japan, the Institute of Electrostatics, Japan, the Institute of Engineers on Electrical Discharges in Japan, and IEEE, USA.

Wibowo Sulistio (Member) is a graduate student of In-

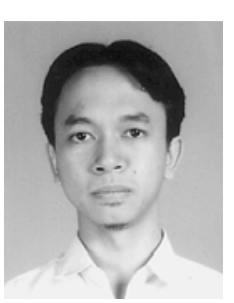
terdiciplinary Graduate School of Engineering Sciences, Kyushu University, from Indonesia. $\mathrm{He}$ is engaged in the study of application of FGM to power engineering. He is a member of the Institute of Electrical Engineers of Japan and the Instutute of Engineers on Electrical Discharges in Japan.

Shin-ichiro Tsuru (Member) is a research staff, Kyushu

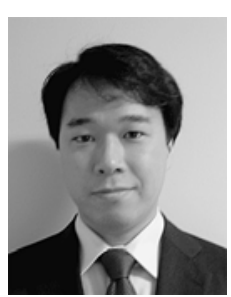
Electric Power Co. Inc., and engaged in research and development of electrical insulation for power apparatus. He is a member of the Institute of Electrical Engineers of Japan, Institute of Engineers on Electrical Discharge in Japan.

Masanori Hara (Member) is a Professor of Faculty of In-

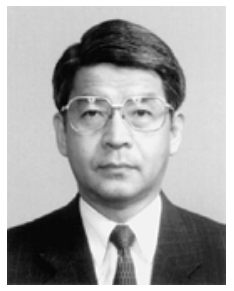
formation Science and Electrical Engineering, Kyushu University, Japan. He is engaged in researches on electric power engineering, HV pulsed power engineering, superconductivity and applied electrostatics. Dr. Hara is a member of the Institute of Electrical Engineers of Japan, the Institute of Electrostatics, Japan, the Cryogenic Association of Japan, the Institute of Engineers on Electrical Discharges in Japan and IEEE, USA. 